TRANSACTIONS OF THE

AMERICAN MATHEMATICAL SOCIETY

Volume 355, Number 6, Pages 2439-2451

S 0002-9947(03)03228-8

Article electronically published on January 14, 2003

\title{
STATIONARY SETS FOR THE WAVE EQUATION IN CRYSTALLOGRAPHIC DOMAINS
}

\author{
MARK L. AGRANOVSKY AND ERIC TODD QUINTO
}

\begin{abstract}
Let $W$ be a crystallographic group in $\mathbb{R}^{n}$ generated by reflections and let $\Omega$ be the fundamental domain of $W$. We characterize stationary sets for the wave equation in $\Omega$ when the initial data is supported in the interior of $\Omega$. The stationary sets are the sets of time-invariant zeros of nontrivial solutions that are identically zero at $t=0$.

We show that, for these initial data, the $(n-1)$-dimensional part of the stationary sets consists of hyperplanes that are mirrors of a crystallographic group $\tilde{W}, W<\tilde{W}$. This part comes from a corresponding odd symmetry of the initial data.

In physical language, the result is that if the initial source is localized strictly inside of the crystalline $\Omega$, then unmovable interference hypersurfaces can only be faces of a crystalline substructure of the original one.
\end{abstract}

\section{Formulation of the Problem AND MAin Results}

Let $\Omega$ be a convex bounded polyhedron in $\mathbb{R}^{n}$, which is the fundamental domain of a crystallographic group $W$ that is generated by orthogonal reflections around a finite number of hyperplanes.

Let us consider the following initial boundary value problem for the wave equation in $\Omega$ with Dirichlet boundary conditions:

$$
\begin{aligned}
& \left(\partial_{t}^{2}-\Delta\right) u=0 \quad \text { in } \quad \Omega, \quad u=u(x, t), x \in \Omega, t>0, \\
& u(x, t)=0, \quad x \in \partial \Omega, t>0, \\
& u(x, 0)=0, \quad x \in \Omega, \\
& \partial_{t} u(x, 0)=f(x), x \in \Omega .
\end{aligned}
$$

Define the stationary set $S_{\Omega}[f]$ as

$$
S_{\Omega}[f]=\{x \in \Omega: u(x, t)=0, \quad \forall t>0\}, \quad u \neq \equiv .
$$

Thus, $S_{\Omega}[f]$ is the set of all points in $\Omega$ where the solution $u(x, t)$ to (1.1) vanishes for all time $t>0$ assuming $u$ is identically zero at $t=0$.

For a separable solution to (1.1),$u(x, t)=\sin \lambda t \cdot \varphi_{\lambda}(t)$, where $\varphi_{\lambda}$ is the eigenfunction of the Laplacian, $\Delta \varphi_{\lambda}=-\lambda^{2} \varphi_{\lambda}$, the stationary set $S_{\Omega}[f]$ coincides with the nodal set $\varphi_{\lambda}^{-1}(0)$. Characterizing nodal sets is a well-known and difficult problem. There is a rich literature on this, and we will mention here only some results

Received by the editors September 4, 2002.

2000 Mathematics Subject Classification. Primary 35L05, 44A12; Secondary 35B05, 35S30.

The first author was supported by the Israel Science Foundation (grant No. 279/02-1).

The second author was partially supported by NSF grants 9877155 and 0200788 . 
relating to our subject. The analytic structure of nodal sets is quite well understood: they consist of ( $n-1)$-dimensional smooth (real-analytic) submanifolds and closed analytic sets of lower dimension (see, e.g., [6], [11], [13], [14], 77, [15] and others).

On the other hand, understanding the global geometry of a single nodal set is a very difficult problem (see, e.g., 16], where the question about existence of closed nodal lines for the second eigenvalue is answered). Nevertheless, and this is the main point of our paper, joint nodal sets in certain situations can be completely described geometrically.

More precisely, we study the case when the stationary sets are intersections of infinitely many nodal sets. This happens, in particular, when the initial velocity $f$ in the wave equation has support strictly in the interior of the domain. It might be expected that in this case the $(n-1)$-dimensional component, if it is nonempty, has a precisely determined geometry.

Indeed, our main result states that the stationary hypersurfaces in crystallographic domains $\Omega=\mathbb{R}^{n} / W$, where $W$ is a crystallographic group generated by reflections, are hyperplanes constituting a crystallographic substructure in $\Omega$. Here is a more precise formulation. We will say $A \Subset B$ if and only if $A$ is a compact subset of the interior of $B$, int $B$.

Theorem 1.1. Let $\Omega$ be a crystallographic domain in $\mathbb{R}^{n}$ generated by the reflection group $W$, and assume $f \in C(\Omega), f \neq 0$, and supp $f$ is a compact subset of int $\Omega$, supp $f \Subset \Omega$. Then the stationary set $S_{\Omega}[f]$ defined in (1.2) is the union

$$
S_{\Omega}[f]=S_{0} \cup V
$$

where $S_{0}$, assuming $S_{0} \neq \emptyset$, is the union of cross sections of $\Omega$ by hyperplanes and $V$ is an analytic set in $\Omega, \operatorname{dim} V<n-1$.

Moreover, there exists a crystallographic group $\tilde{W}$ generated by reflections, $W$ being a subgroup (of finite index) of $\tilde{W}$, such that $S_{0}$ defines a partition of $\Omega$ into a finite number of fundamental polyhedra of the group $\tilde{W}$.

In this case, the initial data $f$ must be skew-symmetric with respect to reflections through the hyperplanes in $S_{0}$.

Let us briefly comment on the result. Brüning [9] characterized the compact sets in the plane with piecewise smooth boundary with the following property: there are an infinite number of eigenfunctions of the Laplacian on the set with common nodal curves that are line segments. His result shows that the only planar polygons with this property are rectangles, equilateral triangles and triangles with the angles $\pi / 4, \pi / 4, \pi / 2$ and $\pi / 6, \pi / 3, \pi / 2$ (and sets made up from them). Note that the listed polygons are just fundamental domains of crystallographic groups generated by reflections. Our result for $n=2$ states that common nodal lines for infinite families of eigenfunctions in these polygons are always straight lines, provided the eigenfunctions appear as projections on eigenspaces of a function $f$ with $\operatorname{supp} f \Subset \operatorname{int} \Omega$. Note that, in general, for these polygons, nodal curves for individual eigenfunctions can be very complicated with many nonlinear curves, including closed curves.

This paper continues a series of works by the authors on stationary sets for the wave equation with compactly supported initial data. For the problem (1.1) with $\Omega=\mathbb{R}^{2}$ and $f \in C_{c}\left(\mathbb{R}^{2}\right)$ (continuous functions with compact support), the stationary sets were completely described in [2]. They have reflection symmetry 
and consist, up to finite sets, of straight lines constituting a Coxeter system (equiangular configuration of straight lines through one point). In higher dimensions, $n>2$, the stationary sets $S[f]$ in $\mathbb{R}^{n}$ were described for the case of finitely supported distributions $f$ [4], and the general case of compactly supported initial data $f$ is still open, but we have conjectured [3] that the result is analogous to the plane case, namely, the $(n-1)$-dimensional parts of stationary sets are cones. Articles such as [1], 5] provide insight into the general case.

The general idea of the proof of Theorem 1.1 is related to results from [2], 3], [4] and is the following. The first step is to extend the problem to the whole space $\mathbb{R}^{n}$ by reflections around the faces of the fundamental polyhedron $\Omega$, which results in a tiling of $\mathbb{R}^{n}$. Then we relate the stationary sets to the kernel of a spherical Radon type transform. A crucial step is a local symmetry principle that we derive from the microlocal analysis of the spherical transform. This and arguments of irrational wrapping type help us analyze $W$-orbits of tangent planes to stationary surfaces and prove that the hypersurfaces are hyperplanes. The final conclusion follows from the reflection principle for the wave equation.

\section{Stationary SETS AND THE SPHERICAL MEANS}

Consider the tiling of $\mathbb{R}^{n}$ resulting from reflections of the fundamental polyhedron $\Omega$ through its faces, and extend $f$ to $\mathbb{R}^{n}$ by odd symmetry: $f\left(\sigma_{1} \ldots \sigma_{k} x\right)=$ $(-1)^{k} f(x), x \in \Omega$, where each element $\sigma_{i} \in W$ is a reflection. It easily follows from the definition of fundamental domain that the extended function is well defined. Let us call the extended function the $W$-extension of $f$.

Using this extension, from now on, we assume that $f$ is defined in $\mathbb{R}^{n}$ and is skew-symmetric with respect to any reflection $\sigma \in W, f \circ \sigma=-f$. Let us consider the following initial value problem in $\mathbb{R}^{n}$ :

$$
\begin{aligned}
& \left(\partial_{t}^{2}-\Delta\right) u=0, \quad u=u(x, t), x \in \mathbb{R}^{n}, t>0, \\
& u(x, 0)=0, \quad x \in \mathbb{R}^{n}, \\
& \partial_{t} u(x, 0)=f(x), \quad x \in \mathbb{R}^{n},
\end{aligned}
$$

where $f \in C\left(\mathbb{R}^{n}\right)$ and $f$ has the following properties:

1) $C \cap \operatorname{supp} f \Subset \operatorname{int} C$ for any open cell $C$ of the group $W$ (i.e., $C=w(\Omega)$ for some $w \in W)$.

2) $f \circ \sigma=-f$ for any reflection $\sigma \in W$.

By the symmetry principle for the wave equation, the solution $u(x, t)$ to (2.1) possesses the same symmetry $u(\sigma x, t)=-u(x, t), x \in \mathbb{R}^{n}, t>0$, for any reflection $\sigma \in W$. Moreover, by (1) and 22), the restriction of $u(x, t)$ to the original polyhedron $\Omega$ solves the problem (1.1) with the initial data $\left.f\right|_{\Omega}$.

Now introduce the spherical transform

$$
R f(x, r)=\int_{S(x, r)} f(y) d A(y),
$$

where $S(x, r)=\left\{y \in \mathbb{R}^{n}:\|y-x\|=r\right\}$ and $d A(y)$ is the normalized area measure on the sphere $S(x, r)$.

Lemma 2.1. The following identity holds:

$$
S[f]=\left\{x \in \mathbb{R}^{n}: R f(x, r)=0, \quad \forall r>0\right\} .
$$


The proof of the lemma is given, for instance, in [3], and it directly follows from the Kirchoff-Poisson formula for the solution $u(x, t)$ and from the invertibility of the resulting generalized Volterra-Abel integral equation.

Lemma 2.2. Let $f=\sum_{k=1}^{\infty} c_{k} \varphi_{k}$ be the Fourier decomposition of $\left.f\right|_{\Omega}$ in the space $L^{2}(\Omega)$ into the orthonormal basis of Dirichlet eigenfunctions $\varphi_{k},\left(\Delta+\lambda_{k}^{2}\right) \varphi_{k}=0$, $\lambda_{k} \neq 0$. Then $S[f]=\bigcap_{k=0, c_{k} \neq 0}^{\infty} \tilde{\varphi}_{k}^{-1}(0)$, where $\tilde{\varphi}_{k}$ is the $W$-extension of $\varphi_{k}$ to $\mathbb{R}^{n}$. Furthermore, each $\tilde{\varphi}_{k}$ is real-analytic.

Proof. Note that the extended functions $\tilde{\varphi}_{k}$ are (smooth) eigenfunctions for the Laplace operator in $\mathbb{R}^{n}$, because they vanish on the faces of the polyhedra $w \Omega, w \in$ $W$, and the normal derivatives in adjacent polyhedra agree on the common faces.

The solution $u(x, t)$ to (2.1) is given by the series

$$
u(x, t)=\sum_{k=0}^{\infty} c_{k} \frac{\sin \lambda_{k} t}{\lambda_{k}} \tilde{\varphi}_{k}(x),
$$

which converges in $L^{2}$ on compact subsets of $\mathbb{R}^{n}$. It follows that $u(x, t)=0$ for all $t>0$ is equivalent to $\tilde{\varphi}_{k}(x)=0, k \in \mathbb{N}, c_{k} \neq 0$.

\section{LOCAL SYMMETRY PRINCIPLE (THE SUPPORT THEOREM)}

The symmetry principle for the wave equation says that if a solution $u(x, t)$ vanishes on a hyperplane $S$ for all $t>0$, then $u(x, t)$ and, in particular, the initial data $f(x)=\partial_{t} u(x, 0)$ are odd with respect to the reflection through $S$.

If the zero hypersurface $S$ is not a hyperplane, this strong symmetry principle does not hold. Nevertheless, we will show that a weaker symmetry principle holds for nonlinear surfaces as well.

Definition 3.1. Let $\Pi$ be a hyperplane in $\mathbb{R}^{n}$ and $a \in \Pi$. We say that a set $K \subset \mathbb{R}^{n}$ is locally symmetric with respect to the pair $(\Pi, a)$ (or (,$a)$-locally symmetric) if whenever a point $x_{0} \in K$ gives a local minimum of the distance function $K \ni x \rightarrow$ $\operatorname{dist}(x, a)=\|x-a\|$, the $\Pi$-mirror point $x_{0}^{*}$ belongs to $K$.

Note that this definition of local symmetry does not imply that $K$ is symmetric about $\Pi$ even near $x_{0}$, but only that if $x_{0}$ is a boundary point of $K$ and $K$ is locally to one side of $S\left(a,\left\|x_{0}-a\right\|\right)$ near $x_{0}$, then the reflection of $x_{0}$ in $\Pi$ is also in $K$.

The following theorem, which is equivalent to Theorem 3.2 in [4, is the key microlocal result. Throughout the article, if $S$ is a manifold and $a \in S$, then $T_{a}(S)$ and $N_{a}(S)$ will denote the tangent and normal spaces correspondingly to $S$ at $a$, and $T_{a}^{\circ}(S)=a+T_{a}(S)$ will denote the affine tangent plane.

Theorem 3.2 (Theorem 3.2 in [4). Let $S$ be a real-analytic hypersurface in $\mathbb{R}^{n}$ and $f \in \mathcal{D}^{\prime}\left(\mathbb{R}^{n}\right)$ a distribution in $\mathbb{R}^{n}$ such that $R f(x, r)=0$ for all $x \in S$ and $r>0$. Then, for each $a \in S$, supp $f$ is $\left(T_{a}^{\circ}(S), a\right)$-locally symmetric.

This theorem is a generalization of a theorem of Courant and Hilbert ([12, p. $699 \mathrm{ff}$.$] ), which states that if S=\Pi$ is a hyperplane and $R f(a, r)=0$ for all $a \in \Pi$ and $r>0$, then $f$ is odd about $\Pi$ and so $\operatorname{supp} f$ is globally symmetric about $\Pi$. (Courant and Hilbert's theorem is equivalent, due to Lemma 2.1] to the reflection principle for the wave equation.) For Theorem 3.2, $S$ can be curved, and in this 
case supp $f$ is only locally symmetric about the tangent planes to $S$ in the weak sense of Definition 3.1

Since the statement of this theorem is slightly different in [4], let us outline the main ideas of the proof.

The key point is the regularity property of the operator $R$, which implies cancellation of analytic wavefront sets at points where a sphere, centered at a point $a \in S$, touches the support of $f$. In turn, the cancellation is possible only at mirror points with respect to the tangent plane $T_{a}^{\circ}(S)$, and this implies that the point that is mirror to the touching point must be in the support as well, and the local symmetry follows.

More precisely, let $x_{0}$ give a local minimum for the distance from $a$ to $\operatorname{supp} f$ and let $r_{0}=\left\|x_{0}-a\right\|$. Let $\mathrm{WF}_{\mathrm{A}}(f)$ be the analytic wavefront set of $f$ [17] and let $\xi_{0}$ be a conormal covector to $S\left(a, r_{0}\right)$ at $x_{0}$. Then, locally near $x_{0}$, supp $f$ lies outside $S\left(a, r_{0}\right)$. By a theorem of Kawai, Kashiwara, and Hörmander [17 about the analytic wavefront set at boundary points of $\operatorname{supp} f,\left(x_{0}, \xi_{0}\right) \in \mathrm{WF}_{\mathrm{A}}(f)$. Let $x_{0}^{*}$ be the reflection of $x_{0}$ in $T_{a}^{\circ}(S)$. By Theorem 3.3 in [4], $x_{0}^{*}$ must be in supp $f$ (if not, $\left(x_{0}, \xi_{0}\right)$ could not be in $\left.\mathrm{WF}_{\mathrm{A}}(f)\right)$. Therefore, $\operatorname{supp} f$ is $\left(T_{a}^{\circ}(S), a\right)$-locally symmetric.

\section{4. $W$-ORBits of the tangent BUndLe $T(S[f])$}

In the sequel, $S$ will denote a connected real-analytic open $(n-1)$-submanifold of $S[f]$. Our aim in this section is to analyze the behavior of the pairs $\left(T_{a}^{\circ}(S), a\right), a \in S$, under the $W$-action.

4.1. Preparations. First, we use the Bieberbach theorem [10] (actually the definition in some books) that states that the crystallographic group $W$ contains a lattice subgroup $\mathbb{Z}\left[h_{1}, \cdots, h_{n}\right]$ generated by $n$ linearly independent vectors $h_{i}$ :

$$
\mathbb{Z}\left[h_{1}, \ldots, h_{n}\right]=\left\{\sum_{i=1}^{n} k_{i} h_{i}: k_{i} \in \mathbb{Z}\right\} .
$$

From now on, the basis $h_{1}, \cdots, h_{n}$ is fixed.

Lemma 4.1. Assume that $S$ is not contained in a hyperplane. Then there exist a point $a \in S$ and a vector $\tau \in T_{a}(S)$,

$$
\tau=\tau_{1} h_{1}+\cdots+\tau_{n} h_{n}, \quad \tau_{i} \in \mathbb{R},
$$

such that the coordinates $\tau_{i}$ are linearly independent over the field $\mathbb{Q}$ of rational numbers.

Proof. Pick an arbitrary point $a \in S$. If the conclusion of the lemma is not true, then for each vector $\tau \in T_{a}(S), \tau=\sum_{i=1}^{n} \tau_{i} h_{i}$, there are integers $r_{i}$ such that

$$
\sum_{i=1}^{n} r_{i} \tau_{i}=0
$$

Thus, the linear space $T_{a}(S)$ is covered by the countable family of subspaces

$$
T_{a}(S) \cap\left\{\sum_{i=1}^{n} \tau_{i} h_{i} \in \mathbb{R}^{n}: \sum_{i=1}^{n} r_{i} \tau_{i}=0\right\}, \quad r_{i} \in \mathbb{Z},
$$


and this is possible only if $T_{a}(S)$ coincides with one of these subspaces, i.e.,

$$
T_{a}(S)=\left\{\sum_{i=1}^{n} \tau_{i} h_{i} \in \mathbb{R}^{n}: \sum_{i=1}^{n} r_{i} \tau_{i}=0\right\},
$$

for some $\left(r_{1}, \ldots, r_{n}\right) \in \mathbb{Z}^{n}$.

There are only countably many such linear spaces, and since the tangent space $T_{a}(S)$ depends on $a \in S$ continuously and $S$ is connected, then $T_{a}(S)$ is a fixed hyperplane for all $a \in S$, and $S$ is contained in a hyperplane.

Proposition 4.2 (Kronecker, cf. [8, Ch.7,1.3, Prop.7]). Let $\theta_{1}, \ldots, \theta_{n-1} \in \mathbb{R}$. Then the vectors

$$
\left(k_{1}+k_{n} \theta_{1}, \ldots, k_{n-1}+k_{n} \theta_{n-1}\right), \quad k_{i} \in \mathbb{Z},
$$

are dense in $\mathbb{R}^{n-1}$ if and only if the numbers $\theta_{1}, \ldots, \theta_{n-1}, 1$ are linearly independent over $\mathbb{Q}$.

In fact, we will need a bit stronger version of Kronecker's theorem:

Corollary 4.3. If $\theta_{1}, \ldots, \theta_{n-1}, 1$ are linearly independent over $\mathbb{Q}$, then the vectors (4.1) are dense in $\mathbb{R}^{n-1}$ when $\left(k_{1}, \ldots, k_{n}\right) \in \mathbb{Z}^{n}$ and $k_{n} \geq 0$.

Proof. Denote by $V_{+}$the semigroup of all vectors (4.1) with $k_{n} \geq 0$, and let $V_{-}=$ $-V_{+}$.

If $V_{+}$is not dense in $\mathbb{R}^{n-1}$, then $V_{-}$is not dense, either, and there exists an open ball $B=B(b, R), R>0$, that contains no element from the closure cl $V_{-}$. By Proposition 4.2, $\mathrm{cl} V_{+} \cup \mathrm{cl} V_{-}=\mathbb{R}^{n-1}$, and therefore $B \subset \mathrm{cl} V_{+}$.

From the definition (4.1) we have $\mathbb{Z}^{n-1}+\operatorname{cl} V_{+} \subset \mathrm{cl} V_{+}$, and in particular, $\mathbb{Z}^{n-1} \subset$ $\operatorname{cl} V_{+}$. Also $B+\operatorname{cl} V_{+} \subset \operatorname{cl} V_{+}+\operatorname{cl} V_{+} \subset \operatorname{cl} V_{+}$, because $\operatorname{cl} V_{+}$is a semigroup. Therefore, for any $s \in \mathbb{N}$,

$$
\mathbb{Z}^{n-1}+s b+B(0, s R)=\mathbb{Z}^{n-1}+s B=\mathbb{Z}^{n-1}+\underbrace{B+\cdots+B}_{\mathrm{s} \text { times }} \subset \operatorname{cl} V_{+} .
$$

Write $s b=[s b]+\{s b\}$, where $[s b]$ and $\{s b\}$ are the vectors of integer and fractional parts of the coordinates, respectively. Since $\mathbb{Z}^{n-1}+[s b]=\mathbb{Z}^{n-1}$, it follows from the above inclusion that

$$
\{s b\}+B(0, s R) \subset \mathrm{cl} V_{+}
$$

and, since $\{s b\} \in[0,1]^{n-1}$ and $s \in \mathbb{N}$ is arbitrary, we see that $\operatorname{cl} V_{+}=\mathbb{R}^{n-1}$.

Lemma 4.4. Let $X, Y$ be linear normed vector spaces with $\operatorname{dim} X=n, \operatorname{dim} Y=$ $n-1$, and let $P: X \rightarrow Y$ be a linear operator with one-dimensional kernel

$$
\text { ker } P=\mathbb{R} \tau, \quad \tau \in X \backslash\{0\} .
$$

Fix a basis $h_{1}, \ldots, h_{n} \in X$ and define the half-lattice

$$
\mathbb{Z}_{+}[h]=\left\{\sum_{j=1}^{n} k_{j} h_{j}: k_{j} \in \mathbb{Z}, k_{n} \geq 0\right\} .
$$

If the coordinates $\tau_{1}, \ldots, \tau_{n}$ of $\tau$ in the basis $\left\{h_{i}\right\}$ are linearly independent over $\mathbb{Q}$, then the image $P\left(\mathbb{Z}_{+}[h]\right)$ is dense in $Y$. 
Proof. Let $v_{i}=P\left(h_{i}\right), i=1, \ldots, n$. Then

$$
\sum_{j=1}^{n} \tau_{j} v_{j}=P(\tau)=0
$$

and since all $\tau_{i} \neq 0$ we can write

$$
v_{n}=\theta_{1} v_{1}+\cdots+\theta_{n-1} v_{n-1},
$$

where $\theta_{i}=-\tau_{i} / \tau_{n}$.

The condition for $\tau_{i}$ implies that $\theta_{1}, \ldots, \theta_{n-1}, 1$ are linearly independent over $\mathbb{Q}$, and by Corollary 4.3 the vectors

$$
x=P\left(\sum_{j=1}^{n} k_{j} h_{j}\right)=\sum_{j=1}^{n} k_{j} v_{j}=\sum_{j=1}^{n-1}\left(k_{j}+k_{n} \theta_{j}\right) v_{j}
$$

are dense in $Y$, where $k_{j}$ runs over $\mathbb{Z}$ and $k_{n} \geq 0$. More precisely, Corollary 4.3 gives denseness in $\mathbb{R}^{n-1}$ of vectors $\left(k_{1}+k_{n} \theta_{1}, \ldots, k_{n-1}+k_{n} \theta_{n-1}\right) \in R^{n-1}$ and then the denseness of vectors $x$ in $Y$ follows because the system $v_{1}, \cdots, v_{n-1}$ is a basis in $Y$. Indeed, if $\sum_{i=1}^{n-1} \alpha v_{i}=0$ for some real $\alpha_{i}$, then $\sum_{i=1}^{n-1} \alpha_{i} h_{i} \in \operatorname{ker} P$. So $\left(\alpha_{1}, \cdots, \alpha_{n-1}, 0\right)=t\left(\tau_{1}, \cdots, \tau_{n}\right)$ for some $t \in \mathbb{R}$, and $\tau_{n} \neq 0$ implies all $\alpha_{i}=0$.

4.2. A geometric construction. In what follows $K$ is a fixed compact set in $\mathbb{R}^{n}$. Fix the point $a \in S$ and the vector $\tau \in T_{a}(S)$ in accordance with Lemma 4.1 Possibly using a rotation, we can normalize $\tau$ by

$$
\|\tau\|=1, \quad \tau_{n}>0 .
$$

Choose a unit normal vector $e \in N_{a}(S)$.

We can assume that

$$
\max _{x \in K}(x, \tau)=0
$$

Denote by

$$
Q=\left\{x \in \mathbb{R}^{n}:(x, \tau)=0\right\}
$$

the supporting hyperplane. Let $K_{0}=K \cap Q$ be the portion of $K$ in $Q$.

Choose a real number $d_{0}$ so that

$$
\max _{x \in K_{0}}(x, e)<d_{0}
$$

and define the hyperplane

$$
\Pi_{0}=\left\{x \in \mathbb{R}^{n}:(x, e)=d_{0}\right\} .
$$

Finally, choose two closest points $x_{0} \in K_{0}$ and $p_{0} \in \Pi_{0}$ :

$$
\left\|x_{0}-p_{0}\right\|=\operatorname{dist}\left(K_{0}, \Pi_{0}\right) .
$$

Since the hyperplanes $Q$ and $\Pi_{0}$ are orthogonal and $x_{0} \in Q$, the point $p_{0}$ belongs to both hyperplanes, $p_{0} \in Q \cap \Pi_{0}$. Also,

$$
\operatorname{dist}\left(K_{0}, \Pi_{0}\right)=d_{0}-\max _{x \in K_{0}}(x, e)>0 .
$$


4.3. The supporting plane $Q$ as a limit of supporting spheres. Let $P_{Q}$ : $\mathbb{R}^{n} \rightarrow Q$ be the orthogonal projection. In this subsection we construct, using the notation and preparations made in subsections 4.1 and 4.2, a sequence of spheres having the following properties:

1) they support (touch) the prescribed compact set $K$;

2 ) they converge to the supporting hyperplane $Q$;

3) the $P_{Q}$-projections of centers of the spheres converge to the point $p_{0} \in Q$, defined in 4.5.

Because ker $P_{Q}=\mathbb{R} \tau$ and since the vector $\tau$ is chosen in accordance with Lemma 4.1, i.e., its coordinates $\tau_{i}$ with respect to the basis $h_{i}$ are linearly independent over $\mathbb{Q}$, Lemma 4.4 applies to the operator $P_{Q}$ and yields that $P_{Q}$ takes the half-lattice $Z_{+}[h]$ onto a dense set in the hyperplane $Q$.

In particular, if one fixes a natural number

$$
M>\left|(a)_{n}\right|,
$$

where $(a)_{n}$ is the $n^{\text {th }}$ coordinate of the point of tangency $a$ in the basis $h_{j}$, then there exists an infinite sequence $t_{m} \in \mathbb{Z}_{+}[h]$ such that

$$
P_{Q}\left(t_{m}\right) \rightarrow p_{0}-P_{Q}\left(a+M h_{n}\right), \quad m \rightarrow \infty,
$$

where $p_{0}$ is defined in (4.5).

If we let

$$
a_{m}=a+t_{m}+M h_{n},
$$

then

$$
\lim _{m \rightarrow \infty} P_{Q}\left(a_{m}\right)=p_{0} .
$$

The formula (4.7) defines a translation $w_{m}: a \rightarrow a_{m}$ that belongs to the group $W$. So $a_{m}$ is in the $W$-orbit of the point $a$.

The sequence $a_{m}$ is unbounded because it is infinite and discrete. It is discrete because it belongs to the lattice $Z_{+}[h]$. So passing to a subsequence we can assume that $\left\|a_{m}\right\| \rightarrow \infty$ as $m \rightarrow \infty$.

Now represent $a_{m}$ as the direct sum

$$
a_{m}=P_{Q}\left(a_{m}\right) \oplus\left(a_{m}, \tau\right) \tau .
$$

The first summand converges and hence is bounded; therefore $\left\|\left(a_{m}, \tau\right)\right\| \rightarrow \infty$ as $m \rightarrow \infty$. If $a_{m}=\left\|a_{m}\right\| \xi_{m}$ (so $\left\|\xi_{m}\right\|=1$ ), then, dividing (4.8) by $\left\|a_{m}\right\|=$ $\left(\left\|P_{Q}\left(a_{m}\right)\right\|^{2}+\left|\left(a_{m}, \tau\right)\right|^{2}\right)^{1 / 2}$ and letting $m \rightarrow \infty$, we obtain

$$
\xi_{m} \rightarrow \pm \tau, \quad m \rightarrow \infty .
$$

However, $\xi_{m} \rightarrow-\tau$ is impossible because the $n$th coordinates, in the basis $h_{i}$, of the vectors $\tau$ and $\xi_{m}$ have opposite signs: $\tau_{n}>0$ by construction, and $\left\|a_{m}\right\|\left(\xi_{m}\right)_{n}=\left(t_{m}\right)_{n}+(a)_{n}+M>\left(t_{m}\right)_{n} \geq 0$ because $t_{m} \in \mathbb{Z}_{+}[h]$ and by the choice of $M$ in (4.6).

Thus,

$$
a_{m} /\left\|a_{m}\right\|=\xi_{m} \rightarrow \tau, \quad m \rightarrow \infty .
$$

Lemma 4.5. Denote $r_{m}=\operatorname{dist}\left(a_{m}, K\right)$ and let $x_{m} \in K$ be such that $\left\|x_{m}-a_{m}\right\|=$ $r_{m}$. Then the sequence $x_{m}$ converges to the point $x_{0}$ defined in subsection 4.2 
Proof. It suffices to prove that $x_{0}$ is the only limit point of the sequence $x_{m}$. Let $\tilde{x}_{0}$ be a limit point, $\tilde{x}_{0}=\lim _{k \rightarrow \infty} x_{m_{k}}$. We can assume, by renumbering, that $x_{m} \rightarrow \tilde{x}_{0}$ as $m \rightarrow \infty$.

Clearly, $\tilde{x}_{0} \in K$, because $x_{m_{k}} \in K$ and $K$ is closed. We now show that $\tilde{x}_{0} \in$ $K \cap Q=K_{0}$. Indeed, for any $x \in K$,

$$
\left\|x_{m}-a_{m}\right\|^{2}=r_{m}^{2} \leq\left\|x-a_{m}\right\|^{2}
$$

or, equivalently,

$$
\left(x, a_{m}\right) \leq\left(x_{m}, a_{m}\right)+(1 / 2)\left(\|x\|^{2}-\left\|x_{m}\right\|^{2}\right) .
$$

Dividing both sides by $\left\|a_{m}\right\|$ and letting $m \rightarrow \infty$, so $\left\|a_{m}\right\| \rightarrow \infty$, we obtain from (4.9):

Thus,

$$
(x, \tau) \leq\left(\tilde{x}_{0}, \tau\right)
$$

$$
\left(\tilde{x}_{0}, \tau\right)=\max _{x \in K}(x, \tau)=0
$$

and hence $\tilde{x}_{0} \in Q$ by the definition of the hyperplane $Q$. Geometrically, what we have checked is that the spheres $S\left(a_{m}, r_{m}\right)$ touching the compact $K$ tend to the supporting hyperplane $Q$ when the centers $a_{m}$ go to infinity.

Note that $\left(x_{m}, \tau\right) \leq 0$ by (4.2), because $x_{m} \in K$. On the other hand, $\left(a_{m}, \tau\right) \rightarrow$ $+\infty$ as $m \rightarrow \infty$, and therefore $\exists m_{0} \in \mathbb{N}$ such that $\left(a_{m}, \tau\right)>0$ for $m \geq m_{0}$. Thus, for such $m, x_{m}$ and $a_{m}$ are on opposite sides of the hyperplane $Q$, and therefore the open ball $B\left(a_{m}, r_{m}\right)$ meets $Q$. Hence its intersection with $Q$ coincides with the nonempty intersection of $Q$ and another open ball centered on $Q$ :

$$
B\left(a_{m}, r_{m}\right) \cap Q=B\left(p_{m}, s_{m}\right) \cap Q,
$$

where $p_{m}=P_{Q}\left(a_{m}\right)$.

By minimality of $r_{m}$, no open ball $B\left(a_{m}, r_{m}\right)$ contains points from $K$, and since $B\left(p_{m}, s_{m}\right) \cap Q \subset B\left(a_{m}, r_{m}\right)$, the ball $B\left(p_{m}, s_{m}\right) \cap Q$ is disjoint from $K$. Therefore $\left\|x_{0}-p_{m}\right\| \geq s_{m}$ and $\left\|\tilde{x}_{0}-p_{m}\right\| \geq s_{m}$, because $x_{0}, \tilde{x}_{0} \in K_{0} \subset K$. In particular, the sequence $s_{m}$ is bounded (because $p_{m}$ converges to $p_{0}$ ), and passing to a subsequence we can assume that $s_{m} \rightarrow s_{0}$ as $m \rightarrow \infty$. Taking limits, we obtain

$$
\left\|x_{0}-p_{0}\right\| \geq s_{0}, \quad\left\|\tilde{x}_{0}-p_{0}\right\| \geq s_{0} .
$$

On the other hand, for $m \geq m_{0}$ we have

$$
\begin{aligned}
\left\|P_{Q}\left(x_{m}\right)-a_{m}\right\|^{2} & =\left\|x_{m}-\left(x_{m}, \tau\right) \tau-a_{m}\right\|^{2} \\
& =\left\|x_{m}-a_{m}\right\|^{2}+\left(x_{m}, \tau\right)\left(2\left(a_{m}, \tau\right)-\left(x_{m}, \tau\right)\right) \leq r_{m}^{2},
\end{aligned}
$$

because $\left\|x_{m}-a_{m}\right\|=r_{m}, \quad\left(x_{m}, \tau\right) \leq 0$ and $\left(a_{m}, \tau\right)>0$.

So, $P_{Q}\left(x_{m}\right)$ belongs to the closed ball $\bar{B}\left(a_{m}, r_{m}\right)$, and since it belongs to $Q$ we have $P_{Q}\left(x_{m}\right) \in \bar{B}\left(p_{m}, s_{m}\right)$, i.e., $\left\|P_{Q}\left(x_{m}\right)-p_{m}\right\| \leq s_{m}$. Letting $m \rightarrow \infty$, we conclude that

$$
\left\|\tilde{x}_{0}-p_{0}\right\| \leq s_{0}
$$

Here we have taken into account that $\tilde{x}_{0} \in Q$ and therefore $P_{Q}\left(\tilde{x}_{0}\right)=\tilde{x}_{0}$. Together with (4.10) it gives

$$
\left\|\tilde{x}_{0}-p_{0}\right\|=s_{0} .
$$

To complete the proof, recall that, by the construction in 4.3, the vector $p_{0}-x_{0}$ is proportional to the vector $e$ (normal to $\Pi_{0}$ ): $p_{0}-x_{0}=\alpha e$, where the coefficient $\alpha=\operatorname{dist}\left(K_{0}, \Pi_{0}\right)$. Using (4.11), we have $p_{0}-\tilde{x}_{0}=s_{0} \eta$ where $\|\eta\|=1$. 
From the first inequality in (4.10), we have $s_{0} \leq \alpha$. Then, using the fact that $\tilde{x}_{0} \in K_{0}$ and $p_{o} \in \Pi_{0}$, we obtain

$$
s_{0} \leq \alpha=\operatorname{dist}\left(K_{0}, \Pi_{0}\right) \leq \operatorname{dist}\left(\tilde{x}_{0}, \Pi_{0}\right)=\left(p_{0}-\tilde{x}_{0}, e\right)=s_{0}(\eta, e) \leq s_{0} .
$$

This yields $\eta=e$ and $\alpha=s_{0}$, i.e., $\tilde{x}_{0}=x_{0}$. So, $x_{m} \rightarrow x_{0}$.

\subsection{Dense $W$-orbits and local symmetry.}

Lemma 4.6. Let $\Omega$ be a fundamental domain of the group $W$, and let $K$ be a compact set strictly inside $\Omega, K \Subset \Omega$. Define

$$
\tilde{K}=\bigcup_{w \in W} w(K) .
$$

Let $S$ be a real-analytic connected hypersurface in $\mathbb{R}^{n}$. Suppose that $\tilde{K}$ is locally symmetric with respect to any pair $\left(T_{a}^{\circ}(S), a\right), s \in S$. Then $\tilde{K}=\emptyset$ unless $S$ is a hyperplane.

Proof. Suppose that $\tilde{K} \neq \emptyset$ (and therefore $K \neq \emptyset$ ) and $S$ is not a hyperplane. We apply the constructions and the results of the previous subsections 4.14.4.3 to the compact $K$. Let $a \in S$ and $\tau \in T_{a}(S)$ be as in Lemma 4.1. and $e \in$ $N_{a}(S), Q, \Pi_{0}, x_{m}, a_{m}, x_{0}, p_{0}$ as in subsections 4.2 and 4.3

First of all, applying a translation, we can assume that the condition (4.2) is fulfilled for the compact $K$. In addition to the condition (4.3) for the number $d_{0}$, we require

$$
\max _{x \in K_{0}}(x, e)<d_{0}<\rho / 2+\max _{x \in K_{0}}(x, e),
$$

where $\rho=\operatorname{dist}(K, \partial \Omega)$. Note that $\rho>0$ because $K \Subset \Omega$.

The points $a_{m}=w_{m}(a)$ in (4.7) belong to the $W$-orbit of the point $a$, and since $\tilde{K}$ is $W$-invariant and $\left(T_{a}^{\circ}(S), a\right)$-locally symmetric, then $\tilde{K}$ is locally symmetric with respect to any pair $\left(\Pi_{m}, a_{m}\right)$, where $\Pi_{m}=T_{a_{m}}^{\circ}\left(S_{m}\right)$ and $S_{m}=w_{m}(S)$.

Because $K$ is open and closed in $\tilde{K}$, the function $\tilde{K} \ni x \rightarrow\left\|x-a_{m}\right\|$ attains a local minimum $r_{m}$ at the point $x_{m}$ and, by local symmetry, Theorem [3.2, the $\left(\Pi_{m}, a_{m}\right)$-mirror point $x_{m}^{*}$ belongs to $\tilde{K}$.

It follows that the $\Pi_{0}$-symmetric point $x_{0}^{*}$ is in $\tilde{K}$. Indeed, since $P_{Q}$ is the orthogonal projection along the vector $\tau$, orthogonal to $e$, then $\left(a_{m}, e\right)=\left(p_{m}, e\right)$ (recall that $p_{m}=P_{Q}\left(a_{m}\right)$ ) and therefore the hyperplane $\Pi_{m}$ is defined by the equation $(x, e)=d_{m}$ where $d_{m}=\left(p_{m}, e\right)$.

The $\Pi_{m}$-mirror point $x_{m}^{*}=x_{0}+2\left(d_{m}-\left(x_{0}, e\right)\right) e \in \tilde{K}$. By Lemma 4.5 $x_{m} \rightarrow x_{0}$, and also $p_{m} \rightarrow p_{0}$. So on taking the limit we have the $\Pi_{0}$-mirror point

$$
x_{0}^{*}=x_{0}+2\left(d_{0}-\left(x_{0}, e\right)\right) e \in \tilde{K}, \quad d_{0}=\left(p_{0}, e\right) .
$$

Furthermore, the right inequality in (4.12) implies

$$
\left\|x_{0}^{*}-x_{0}\right\|=2\left|d_{0}-\left(x_{0}, e\right)\right|<\rho,
$$

and since $x_{0} \in K \subset \Omega$, then $x_{0}^{*}$ must be inside $\Omega$ by the definition of $\rho$, and so $x_{0}^{*} \in \Omega \cap \tilde{K}=K$.

We will now show $x_{0}^{*}$ is not in $K$ by showing $x_{0}^{*}$ is above $K_{0}$. This will finish the proof. 
Moreover, $x_{0}^{*} \in Q \cap K=K_{0}$ because $x_{0} \in Q$, and the hyperplane $Q$ is orthogonal to $\Pi_{0}$, and hence $Q$ is invariant under reflection in $\Pi_{0}$. From this and from the left inequality in (4.12) we have

$$
\left(x_{0}^{*}, e\right) \leq \max _{x \in K_{0}}(x, e)<d_{0} .
$$

However, the same inequality in (4.12) implies

$$
\left(x_{0}^{*}, e\right)=\left(x_{0}, e\right)+2\left(d_{0}-\left(x_{0}, e\right)\right)=2 d_{0}-\left(x_{0}, e\right) \geq 2 d_{0}-\max _{x \in K_{0}}(x, e)>d_{0} .
$$

This contradiction proves that if $S$ is not a hyperplane, then $\tilde{K}=\emptyset$.

\section{Proof of Theorem 1.1}

Recall that we deal with the wave equation (2.1) in the whole space $\mathbb{R}^{n}$, and the initial data $f$ is obtained by the $W$-extension in section 11. Correspondingly, the stationary set $S[f]$ is obtained from $S_{\Omega}[f]$ (see (1.2)) by applying the group $W$ and adding hyperplanes that bound all the polyhedra $w(\Omega), w \in W$. We call these hyperplanes $W$-mirrors; they are obtained from the system of hyperplanes generating the group $W$ by applying elements from $W$, and each $W$-mirror consists of fixed points of some reflection in $W$.

Denote $K=\Omega \cap \operatorname{supp} f$. Since $f \neq 0$, we have $K \neq \emptyset$. Because $f$ is skewsymmetric with respect to reflections from the group $W, \tilde{K}=\operatorname{supp} f=\bigcup_{w \in W} w K$.

According to Lemma 2.2, the stationary set

$$
S[f]=\bigcap_{k=0, c_{k} \neq 0}^{\infty} \tilde{\varphi}_{k}^{-1}(0) ;
$$

and since each nodal set $\tilde{\varphi}_{k}(0)$ is a union of a smooth hypersurface and a lowerdimensional analytic set (see Theorem 1.1), the representation

$$
S[f]=S_{0} \cap V
$$

holds, where $S_{0}$ is a smooth hypersurface and $V$ is an analytic set of dimension less than $n-1$.

Theorem 3.2 states that the set $\tilde{K}=\operatorname{supp} f$ is $\left(T_{a}^{\circ}\left(S_{0}\right), a\right)$-locally symmetric for any $a \in S_{0}$. Then Lemma 4.6 implies that any connected smooth hypersurface $S \subset S_{0}$ must be a hyperplane. Therefore $S_{0}$ is a union of hyperplanes.

Denote by $\Sigma$ the system of these hyperplanes, so that

$$
S_{0}=\bigcup_{\pi \in \Sigma} \pi
$$

Since $S_{0} \subset S[f]$, then by the reflection principle, the initial data $f$ is odd with respect to reflections through any hyperplane in $\Sigma$. Also all $W$-mirrors belong to $\Sigma$.

Let $\tilde{W}$ be the group generated by the reflections through the hyperplanes in $\Sigma$. Since $\Sigma$ contains $W$-mirrors, $W \subset \tilde{W}$. By the above property of $f$, each set $S[f], S_{0}$ and $V$ is $\tilde{W}$-invariant, and for this reason $\Sigma$ is a $\tilde{W}$-invariant system of hyperplanes.

We claim that the system $\Sigma$ is locally finite. Indeed, if it is not, then there exists a hyperplane $\pi_{0}$ in $\mathbb{R}^{n}$ that is a limit point of hyperplanes in $\Sigma$ :

$$
\pi_{0}=\lim _{m \rightarrow \infty} \pi_{m}, \quad \pi_{m} \in \Sigma, \quad \pi_{m} \neq \pi_{0} .
$$


By Lemma 2.2 each eigenfunction $\tilde{\varphi}_{k}$ in the Fourier decomposition (2.3) vanishes on $S[f]$ and hence vanishes on each hyperplane $\pi_{m}$. Then $\tilde{\varphi}_{k}$ vanishes on $\pi_{0}$ to infinite order, and $\varphi_{k}=0$ due to real analyticity. This would imply $f=0$, which is not the case.

Now we want to check that the group $\tilde{W}$ is discrete. It suffices to prove that the unit $e$, the identity map, is an isolated point of $\tilde{W}$.

Suppose $w_{m} \rightarrow e$, where $w_{m} \in \tilde{W}$. The convergence is uniform on compact sets. Due to local finiteness of the system $\Sigma$, only a finite number of hyperplanes in $\Sigma$ meet the fundamental polyhedron $\Omega$; therefore $\Omega \backslash S_{0}$ consists of a finite number of disjoint open polyhedra. Let $\Delta$ be one of them, and $c \in \Delta$ a generic point (not a fixed point of any nontrivial isometry of $\Delta$ ). Then $w_{m}(c) \rightarrow c$, and hence $\exists m_{0} \in \mathbb{N}$ such that $w_{m}(c) \in \Delta$ for $m \geq m_{0}$. Since for each $m>m_{0}, w_{m}$ is a composition of $\Sigma$-reflections and the polyhedron $w_{m}(\Delta)$ and $\Delta$ have a common point, $w_{m}(c)$, they must coincide: $w_{m}(\Delta)=\Delta$ for $m \geq m_{0}$.

However, the group of isometries of the polyhedron $\Delta$ is finite, and hence the sequence $w_{m}$ contains only a finite number of distinct elements. Then, since $c$ is generic, $w_{m}(c) \rightarrow c$ implies that $w_{m}=e$ when $m$ is sufficiently large. This means that the group $\tilde{W}$ is discrete.

In addition, the factor-space $\mathbb{R}^{n} / \tilde{W}$ is compact because of the imbedding

$$
\mathbb{R}^{n} / \tilde{W} \hookrightarrow \mathbb{R}^{n} / W \simeq \bar{\Omega} / W
$$

and since $\bar{\Omega}$ is compact.

Thus the group $\tilde{W}$ is uniform (the factor space is compact) and discrete, and therefore is crystallographic (see, e.g., [10, Def. 1.7). Any reflection from $\tilde{W}$ takes the polyhedron $\Delta$ to a polyhedron that is either inside $\Omega$ or outside $\bar{\Omega}$; the polyhedra obtained by subsequent reflections through the faces are mutually disjoint and cover $\mathbb{R}^{n} \backslash S_{0}$.

Therefore $\Delta$ serves as a fundamental polyhedron of the group $\tilde{W}$. By construction, the group $W$ is a subgroup of the group $\tilde{W}$, of finite index $\#(\tilde{W} / W)$, which equals the number of reflections of the fundamental polyhedron $\Delta$ through its faces needed to cover $\Omega$. This completes the proof.

Remark 5.1. The proof is valid for more complicated domains, namely domains that tile $\mathbb{R}^{n}$ and that are made up of unions of fundamental domains of the same crystallographic group. For example, the hexagon $\Omega$ is a union of equilateral triangles, and it tiles the plane. Our theorem is true for the hexagon as long as $f$ is zero near the boundary of $\Omega$ and $f$ is oddly symmetric about each reflection in each diameter of $\Omega$. The proof is as given above, and the extra condition on $f$ is used to ensure that the extension of $f$ to $\mathbb{R}^{2}$ by odd symmetry is consistent (see the start of $\left.\varliminf_{2}\right)$.

\section{ACKNOWLEDGMENTS}

The authors thank Professors Gregory Soifer and Mikhail Katz for stimulating discussions. The second author would like to thank Dr. Mark Varvares of MEEI and Dr. James McIntyre of MGH for their invaluable help as this research began.

\section{REFERENCES}

1. M. Agranovsky, C. Berenstein, and P. Kuchment Approximation by spherical waves in $L^{p}$ spaces, J. Geom. Analysis 6 (1996), 365-383. MR 99c:41038 
2. M. Agranovsky and E. T. Quinto, Injectivity sets for the Radon transform over circles and complete systems of radial functions, J. Funct. Anal. 139 (2), (1996), 383-414. MR 98g:58171

3. M. Agranovsky and E. T. Quinto, Injectivity of the spherical mean operator and related problems, in: Complex Analysis, Harmonic Analysis and Applications (R. Deville et al, eds.), Addison Wesley, London, 1996, pp. 12-36. MR 97m:44004

4. M. Agranovsky and E. T. Quinto, Geometry of stationary sets for the wave equation in $\mathbb{R}^{n}$. The case of finitely supported initial data, Duke Math. J. 107 (2001), 57-84. MR 2001m:35192

5. M. Agranovsky, V. V. Volchkov and L. Zalcman, Conical Uniqueness Sets for the Spherical Radon Transform, Bull. London Math. Soc., 31(1999), 231-236. MR 2000j:44002

6. C. Bar, On nodal sets for Dirac and Laplace operators, Comm. Math. Phys., 188 (1997), 709-721. MR 98g:58179

7. P. Bérard and D. Meyer Inégalités isopérimétriques et applications, Ann. Sci. École Norm. Sup. 15 (1982), 513-542. MR 84h:58147

8. N. Bourbaki, Éléments de Mathématiques, Livre III, Topologie Générale, Actualités Sci. Ind., no 1092, Hermann et cie., Paris, 1947, MR 9:261a

9. J. Brüning, Über Membranen mit speziellen Knotenlinien, Comment. Math. Helv. 55(1980), 13-19. MR 81e:53040

10. L. S. Charlap, Bieberbach Groups and Flat Manifolds. Springer-Verlag, New York, 1986. MR 88j:57042

11. S. Y. Cheng, Eigenfunctions and nodal sets, Comment. Math. Helv., 51 (1976), 43-55. MR 53:1661

12. Courant R. and Hilbert D., Methods of Mathematical Physics, vol. 2, Interscience, New York, 1961. MR 25:4216

13. H. Donnelly and C. Fefferman, Nodal sets of eigenfunctions on Riemannian manifolds, Invent. Math. 93 (1988), 161-183. MR 89m:58207]

14. 3 (1990), 333-353. MR 92d:58209

15. R. Hardt and L. Simon, Nodal sets for solutions of elliptic equations, J. Diff. Geom. 30(1989), 505-522. MR 90m:58031

16. M. Hoffmann-Ostenhof, T. Hoffmann-Ostenhof and N. Nadirashvili, The nodal line of the second eigenfunction of the Laplacian in $\mathbb{R}^{2}$ can be closed, Duke Math. J., 90 (1997), 3, 631-640. MR 98m:35146

17. L. Hörmander, The Analysis of Linear Partial Differential Operators I, Springer-Verlag, New York, 1983. MR 85g:35002a

Bar Ilan University, Ramat Gan, IsRael

E-mail address: agranovs@macs.biu.ac.il

Tufts University, Medford, Massachusetts

E-mail address: todd.quinto@tufts.edu 